\title{
On a Coupled System of Stochastic Itô-Differential and the Arbitrary (Fractional) Order Differential Equations with Nonlocal Random and Stochastic Integral Conditions
}

\author{
A. M. A. El-Sayed ${ }^{\dagger}$ and Hoda A. Fouad ${ }^{*,+}$
}

check for

updates

Citation: El-Sayed, A.M.A.; Fouad, H.A. On a Coupled System of Stochastic Itô-Differential and the Arbitrary (Fractional) Order Differential Equations with Nonlocal Random and Stochastic Integral Conditions. Mathematics 2021, 9, 2571 https://doi.org/10.3390/math9202571

Academic Editors: Snezhana Hristova and Sitnik Sergey

Received: 2 September 2021

Accepted: 11 October 2021

Published: 14 October 2021

Publisher's Note: MDPI stays neutral with regard to jurisdictional claims in published maps and institutional affiliations.

Copyright: (c) 2021 by the authors. Licensee MDPI, Basel, Switzerland. This article is an open access article distributed under the terms and conditions of the Creative Commons Attribution (CC BY) license (https:/ / creativecommons.org/licenses/by/ $4.0 /)$.
Faculty of Science, Alexandria University, Alexandria 21568, Egypt; amasayed@alexu.edu.eg

* Correspondence: hoda.fouad@alexu.edu.eg

+ These authors contributed equally to this work.

\begin{abstract}
The fractional stochastic differential equations had many applications in interpreting many events and phenomena of life, and the nonlocal conditions describe numerous problems in physics and finance. Here, we are concerned with the combination between the three senses of derivatives, the stochastic Itô-differential and the fractional and integer orders derivative for the second order stochastic process in two nonlocal problems of a coupled system of two random and stochastic differential equations with two nonlocal stochastic and random integral conditions and a coupled system of two stochastic and random integral conditions. We study the existence of mean square continuous solutions of these two nonlocal problems by using the Schauder fixed point theorem. We discuss the sufficient conditions and the continuous dependence for the unique solution.
\end{abstract}

Keywords: stochastic processes; Itô-differential equations; random differential equations; stochastic differential equation; coupled system; fractional order derivative; nonlocal stochastic integral conditions

\section{Introduction}

The existence and uniqueness of solutions to stochastic differential equations driven by Brownian motion have been studied by many authors (see [1-3] ).

Also the non-local coupled system was studied by some authors (see for example [4-8] and references therein).

The results are important since they cover non-local generalizations of fractional stochastic differential equations (FSDE), more applications are arising in fields such as heat conduction, electromagnetic theory and dynamic system (see for example $[9,10]$ ).

Many authors have been interested to study the fractional stochastic differential equations see [11-14] and investigate their results all the time.

Let $(\Omega, G, \mu)$ be a probability space see [15].

The motive of this work is to generalize the scope results of A.M.A. El-Sayed [16,17] on the stochastic fractional operators and the solution of non-local coupled systems of stochastic differential equations see $[7,16]$.

Also, we study the existence of solutions of a coupled system of Itô-differential equation and arbitrary (fractional)orders random differential equation subject to two coupled systems of non-local random and stochastic integral conditions. The effect of random functions and data which ensures the continuous dependence of the solution has been proved.

Let $I=[0, T]$ and $X(t ; \omega)=X(t), t \in I, \omega \in \Omega$ be such that $E\left(X^{2}(t)\right)<\infty, t \in I$.

Let $C=C\left(I, L_{2}(\Omega)\right)$ be the class of all mean square (m.s) continuous stochastic processes on $I$ with norm

$$
\|X\|_{C}=\sup _{t \in I}\|X(t)\|_{2}, \quad\|X(t)\|_{2}=\left(E X^{2}(t)\right)^{1 / 2} .
$$


Definition 1. Let $X \in C\left(I, L_{2}(\Omega)\right)$ and $\mu, v \in(0,1]$. The stochastic integral operator of order $v$ is defined by

$$
I^{v} X(t)=\int_{0}^{t} \frac{(t-s)^{v-1}}{\Gamma(v)} X(s) d s
$$

and the stochastic fractional order derivative is defined by

$$
D^{\mu} X(t)=I^{1-\mu} \frac{d X}{d t}
$$

For the properties of stochastic fractional calculus see [16].

Let $\alpha, \beta \in(0,1]$ and $T \geq 1$. Here we prove the existence of solutions $X, Y \in$ $C\left([0, T], L_{2}(\Omega)\right)$ of the two nonlocal fractional coupled system of the two Itô-deferential and arbitrary orders, differential equations

$$
\frac{d X(t)}{d t}=g_{1}(t, Y(\phi(t))), \quad t \in(0, T]
$$

and

$$
\left.d Y(t)=g_{2}\left(t, D^{\alpha} X(t)\right)\right) d W(t), \quad t \in(0, T]
$$

with the stochastic and random integral conditions

$$
X(0)+\int_{0}^{\tau} h_{1}\left(s, D^{\beta} X(s)\right) d W(s)=X_{o}, \quad Y(0)+\int_{0}^{\eta} h_{2}(s, Y(s)) d s=Y_{o}
$$

and the coupled system of the two stochastic and random non-local integral conditions

$$
X(0)+\int_{0}^{\tau} h_{1}(s, Y(s)) d W(s)=X_{o}, \quad Y(0)+\int_{0}^{\eta} h_{2}(s, X(s)) d s=Y_{0}
$$

where $X_{0}$ and $Y_{0}$ are two second order random variables.

The existence of solutions $X, Y \in C\left(I, L_{2}(\Omega)\right)$ of the problems (1)-(3) and (1)-(2) and (4) are proved. The continuous dependence of the unique solutions $X, Y \in C\left(I, L_{2}(\Omega)\right)$ on $X_{o}$ and $Y_{o}, h_{1}$ and $h_{2}$ and the solution $Y \in C\left(I, L_{2}(\Omega)\right)$ on $D^{\alpha} X(t)$ will be studied.

\section{Integral Representations of the Solution}

Consider the following assumptions:

(a1) $\phi: I \rightarrow I, \phi(t) \leq t$ is a continuous on $I$.

(a2) $g_{i}: I \times L_{2}(\Omega) \rightarrow L_{2}(\Omega)$ are (Caratheodory) measurable in $t \in I, \quad \forall X \in L_{2}(\Omega)$ and continuous in $X \in L_{2}(\Omega) \forall t \in I$ and there exist $b_{i}>0$ and two bounded measurable functions $\xi_{i}: I \rightarrow R$ such that

$$
\left\|g_{i}(t, X)\right\|_{2} \leq\left|\xi_{i}(t)\right|+b_{i}\|X(t)\|_{2}, \quad i=1,2 .
$$

(a3) $h_{i}:[0, T] \times L_{2}(\Omega) \rightarrow L_{2}(\Omega)$ are Caratheodory. There exist $c_{i}>0$ and two bounded measurable functions $\sigma_{i}: I \rightarrow R$ such that

$$
\left\|h_{i}(t, X)\right\|_{2} \leq\left|\sigma_{i}(t)\right|+c_{i}\|X(t)\|_{2}, \quad i=1,2 .
$$

(a4) $M=\max \left\{\sup _{t \in I}\left|\xi_{1}(t)\right|, \sup _{t \in I}\left|\xi_{2}(t)\right|\right\}, b=\max \left\{b_{1}, b_{2}\right\}$.

(a5) $K=\max \left\{\sup _{t \in I}\left|\sigma_{1}(t)\right|, \sup _{t \in I}\left|\sigma_{2}(t)\right|\right\}, c=\max \left\{c_{1}, c_{2}\right\}$.

(a6) $(b+c) T+b^{2} T^{2}(2-\alpha)<1$.

Now, operating by $I^{1-\alpha}$ on Equation (1), we obtain

$$
\left.D^{\alpha} X(t)\right)=I^{1-\alpha} \frac{d X}{d t}=I^{1-\alpha} g_{1}(t, Y(\phi(t))) .
$$


Let

$$
u(t)=D^{\alpha} X(t)
$$

then from (1)-(2) we obtain

$$
u(t)=I^{1-\alpha} g_{1}(t, Y(\phi(t))
$$

and

$$
d Y(t)=g_{2}(t, u(t)) d W(t)
$$

Then we have the following lemma

Lemma 1. The solutions of the problems (1)-(3) and (1), (2) and (4) can be given by

$$
\begin{aligned}
& X(t)=X_{0}-\int_{0}^{\tau} h_{1}\left(s, I^{1-\beta} g_{1}(s, Y(\phi(s)))\right) d W(s)+\int_{0}^{t} g_{1}(s, Y(\phi(s))) d s, \\
& Y(t)=Y_{0}-\int_{0}^{\eta} h_{2}(s, Y(s)) d s+\int_{0}^{t} g_{2}\left(s, I^{1-\alpha} g_{1}(s, Y(\phi(s)))\right) d W(s)
\end{aligned}
$$

and

$$
\begin{aligned}
& X(t)=X_{o}-\int_{0}^{\tau} h_{1}(s, Y(s)) d W(s)+\int_{0}^{t} g_{1}(s, Y(\phi(s))) d s \\
& Y(t)=Y_{o}-\int_{0}^{\eta} h_{2}(s, X(s)) d s+\int_{0}^{t} g_{2}\left(s, I^{1-\alpha} g_{1}(s, Y(\phi(s)))\right) d W(s),
\end{aligned}
$$

respectively.

Proof. Integrating the Equations (7) and (9) (see [12-18]) with substitution by (8) and using the non-local conditions (3) and (4) the equivalent between the problem (1)-(3) and the integral representation (10)-(11) and the problem (1), (2) and (4) and the integral representation (12)-(13) can be proved.

\section{Solutions of the Problem (1)-(3)}

Theorem 1. Let the assumptions (a1)-(a6) be satisfied, then the problem (1)-(3) has at least one solution $X, Y \in C$.

Proof. Consider the set $Q_{1}$ such that

$$
Q_{1}=\left\{Y(t) \in L_{2}(\Omega):\|Y(t)\|_{2} \leq r_{1}\right\} \subset C\left(I, L_{2}(\Omega)\right)
$$

and define the mapping $F_{1}$ where

$$
F_{1} Y(t)=Y_{o}-\int_{0}^{\eta} h_{2}(s, Y(s)) d s+\int_{0}^{t} g_{2}\left(s, I^{1-\alpha} g_{1}(s, Y(\phi(s)))\right) d W(s) .
$$


Let $Y \in Q_{1}$, then

$$
\begin{aligned}
\left\|F_{1} Y(t)\right\|_{2} & \leq\left\|Y_{0}\right\|_{2}+\left\|\int_{0}^{\eta} h_{2}(s, Y(s)) d s\right\|_{2}+\left\|\int_{0}^{t} g_{2}\left(s, I^{1-\alpha} g_{1}(s, Y(\phi(s)))\right) d W(s)\right\|_{2} \\
& \leq\left\|Y_{0}\right\|_{2}+\int_{0}^{\eta}\left(\sigma_{2}(s)+c_{2}\|Y\|_{C}\right) d s \\
& +\sqrt{\int_{0}^{t}\left(\xi_{2}(s)+b_{2} \| I^{1-\alpha} g_{1}(s, Y(\phi(s)))\right) \|_{2}^{2} d s} \\
& \leq\left\|Y_{0}\right\|_{2}+\left(K+c r_{1}\right) T+\left(M+\frac{b T^{1-\alpha}\left(M+b r_{1}\right)}{(1-\alpha) \Gamma(1-\alpha)}\right) \sqrt{T} \\
& \leq\left\|Y_{o}\right\|_{2}+K T+M \sqrt{T}+\frac{M b T^{\frac{3}{2}-\alpha}}{\Gamma(2-\alpha)}+\left(c T+\frac{b^{2} T^{\frac{3}{2}-\alpha}}{\Gamma(2-\alpha)}\right) r_{1} \\
& \leq\left\|Y_{o}\right\|_{2}+(K+M) T+M b T^{2}(2-\alpha)+\left(c T+b^{2} T^{2}(2-\alpha)\right) r_{1}=r_{1}
\end{aligned}
$$

where

$$
r_{1}=\frac{\left(\left\|Y_{o}\right\|_{2}+(K+M)\right) T+M b T^{2}(2-\alpha)}{1-c T-b^{2} T^{2}(2-\alpha)},
$$

then $F_{1}: Q_{1} \rightarrow Q_{1}$ and the class $\left\{F_{1} Y(t)\right\}, t \in[0, T]$ is uniformly bounded on $Q_{1}$.

Let $t_{1}, t_{2} \in[0, T]$ such that $\left|t_{2}-t_{1}\right|<\delta$, then

$$
\begin{aligned}
\left\|F_{1} Y\left(t_{1}\right)-F_{1} Y\left(t_{2}\right)\right\|_{2} & =\| \int_{0}^{t_{2}} g_{2}\left(s, I^{1-\alpha} g_{1}(s, Y(\phi(s)))\right) d W(s) \\
& -\int_{0}^{t_{1}} g_{2}\left(s, I^{1-\alpha} g_{1}(s, Y(\phi(s)))\right) d W(s) \|_{2} \\
& =\left\|\int_{t_{1}}^{t_{2}} g_{2}\left(s, I^{1-\alpha} g_{1}(s, Y(\phi(s)))\right) d W(s)\right\|_{2} \\
& \leq \sqrt{\int_{t_{1}}^{t_{2}} g_{2}\left(s, I^{1-\alpha} g_{1}(s, Y(\phi(s)))\right) \|_{2}^{2} d s} \\
& \leq\left(M+b\left(\frac{T^{1-\alpha}\left(M+b\|Y\|_{c}\right)}{\Gamma(2-\alpha)}\right)\right) \sqrt{t_{2}-t_{1}} .
\end{aligned}
$$

This proves the equicontinuity of the class $\left\{F_{1} Y(t)\right\}, t \in[0, T]$ on $Q_{1}$.

Let $Y_{n} \in Q_{1}, \quad Y_{n} \rightarrow Y \quad$ w.p.1, we get (see [15])

$$
\begin{aligned}
& \text { L.i. } m_{n \rightarrow \infty} F_{1} Y_{n}=\operatorname{Li} . m_{n \rightarrow \infty}\left(Y_{0}-\int_{0}^{\eta} h_{2}\left(s, Y_{n}(s)\right) d s+\int_{0}^{t} g_{2}\left(s, I^{1-\alpha} g_{1}\left(s, Y_{n}(\phi(s))\right)\right) d W(s)\right) \\
& =Y_{0}-\int_{0}^{\eta} h_{2}\left(s, \text { L.i.m. } m_{n \rightarrow \infty} Y_{n}(s)\right) d s \\
& +\int_{0}^{t} g_{2}\left(s, I^{1-\alpha} g_{1}\left(s, L . i . m_{n \rightarrow \infty} Y_{n}(\phi(s))\right)\right) d W(s) \\
& =Y_{o}-\int_{0}^{\eta} h_{2}(s, Y(s)) d s+\int_{0}^{t} g_{2}\left(s, I^{1-\alpha} g_{1}(s, Y(\phi(s)))\right) d W(s)=F_{1} Y .
\end{aligned}
$$

This proves that the operator $F_{1}: Q_{1} \rightarrow Q_{1}$ is continuous. Consequently, the closure of $\left\{F_{1} Q_{1}\right\}$ is compact and (see [15]) and integral Equation (11) has a solution $Y \in$ $C\left([0, T], L_{2}(\Omega)\right)$.

Let the set $Q_{2}$ be such that

$$
Q_{2}=\left\{X(t) \in L_{2}(\Omega):\|X(t)\|_{2} \leq r_{2}\right\} \subset C\left([0, T], L_{2}(\Omega)\right)
$$


and define the mapping $F_{2} X$ such that

$$
F_{2} X(t)=X_{o}-\int_{0}^{\tau} h_{1}\left(s, I^{1-\beta} g_{1}(s, Y(\phi(s)))\right) d W(s)+\int_{0}^{t} g_{1}(s, Y(\phi(s))) d s .
$$

Let $X \in Q_{2}$, then

$$
\begin{aligned}
\left\|F_{2} X(t)\right\|_{2} & \leq\left\|X_{o}\right\|_{2}+\sqrt{\int_{0}^{\tau} \| h_{1}\left(s, I^{\left.1-\beta_{g_{1}}(s, Y(\phi(s)))\right) d W(s) \|_{2}^{2} d s}\right.}+\int_{0}^{t}\left\|g_{1}(s, Y(\phi(s)))\right\|_{2} d s \\
& \leq\left\|X_{o}\right\|_{2}+\sqrt{\int_{0}^{\tau}\left(\left|\sigma_{1}(s)\right|+c_{1}\left\|I^{1-\beta} g_{1}(s, Y(\phi(s)))\right\|_{2}\right)^{2} d s}+\int_{0}^{t}\left(\left|\xi_{1}(s)\right|+b_{1}\|Y\|_{C}\right) d s \\
& \leq\left\|X_{o}\right\|_{2}+\left(K+\frac{c T^{1-\beta}\left(M+b r_{1}\right)}{\Gamma(2-\alpha)}\right) \sqrt{T}+\left(M+b r_{1}\right) T \\
& \leq\left\|X_{o}\right\|_{2}+(K+M) T+\frac{c M T^{\frac{3}{2}-\beta}}{\Gamma(2-\alpha)}+\left(\frac{c b T^{\frac{3}{2}-\beta}}{\Gamma(2-\alpha)}+b T\right) r_{1} \\
& \leq\left\|X_{o}\right\|_{2}+(K+M) T+M c T^{2}(2-\beta)+\left(b T+b c T^{2}(2-\beta)\right) r_{1}=r_{2}
\end{aligned}
$$

where

$$
r_{2}=\left\|X_{0}\right\|_{2}+(K+M) T+M c T^{2}(2-\beta)+\left(b T+b c T^{2}(2-\beta)\right) r_{1},
$$

then the class $\left\{F_{2} X(t)\right\}, \quad t \in[0, T]$ is uniformly bounded.

Let $X \in Q_{2} ; \quad t_{1}, t_{2} \in[0, T]$, then

$$
\begin{aligned}
\left\|F_{2} X\left(t_{2}\right)-F_{2} X\left(t_{1}\right)\right\|_{2} & =\left\|\int_{t_{1}}^{t_{2}} g_{1}(s, Y(\phi(s))) d s\right\|_{2} \\
& \leq\left(M+b r_{1}\right)\left(t_{2}-t_{1}\right)
\end{aligned}
$$

then the class $\left\{F_{2} X(t)\right\}, \quad t \in[0, T]$ is equicontinuous.

Let $X_{n} \in Q_{2}$ be such that $X_{n} \rightarrow Y \quad$ w.p.1.

Using Theorem 1 , we get

$$
\begin{aligned}
\operatorname{L.i.m}_{n \rightarrow \infty} F_{2} X_{n} & =\operatorname{L.i.m_{n\rightarrow \infty }}\left(X_{o}-\int_{0}^{\tau} h_{1}\left(s, I^{1-\beta} g_{1}\left(s, Y_{n}(\phi(s))\right)\right) d W(s)+\int_{0}^{t} g_{1}\left(s, Y_{n}(\phi(s))\right) d s\right) \\
& =X_{o}-\int_{0}^{\tau} h_{1}\left(s, L . i . m_{n \rightarrow \infty} I^{1-\beta_{g}} g_{1}\left(s, Y_{n}(\phi(s))\right)\right) d W(s)+\int_{0}^{t} g_{1}\left(s, L . i . m_{n \rightarrow \infty} Y_{n}(\phi(s))\right) d s \\
& =X_{o}-\int_{0}^{\tau} h_{1}\left(s, I^{1-}{ }^{1-} g_{1}(s, Y(\phi(s)))\right) d W(s)+\int_{0}^{t} g_{1}(s, Y(\phi(s))) d s=F_{2} X .
\end{aligned}
$$

Applying Schauder Fixed Point Theorem [15], (10) has a solution $X \in C\left(I, L_{2}(\Omega)\right)$.

\subsection{Uniqueness Theorem}

To discuss the uniqueness of the solution $Y$ of (11) consider the assumptions $\left(a^{*} 1\right)-$ $\left(a^{*} 2\right)$ instead of $(a 1)-(a 2)$ such that

$\left(a^{*} 1\right) g_{i}: I \times L_{2}(\Omega) \rightarrow L_{2}(\Omega) ; \quad i=1,2$ are Caratheodory and satisfy second argument

Lipschitz condition

$$
\left\|g_{i}(t, u(t))-g_{i}(t, v(t))\right\|_{2} \leq b\|u(t)-v(t)\|_{2} .
$$

$\left(a^{*} 2\right) h_{i}: I \times L_{2}(\Omega) \rightarrow L_{2}(\Omega) ; \quad i=1,2$ Caratheodory and satisfy second argument Lipschitz condition

$$
\left\|h_{i}(t, u(t))-h_{i}(t, v(t))\right\|_{2} \leq c\|u(t)-v(t)\|_{2} .
$$

It is clear that the assumptions $\left(a^{*} 1\right)-\left(a^{*} 2\right)$ imply the assumptions $(a 1)-(a 2)$. 
Theorem 2. Let $\left(a^{*} 1\right)-\left(a^{*} 2\right)$ and $(a 3)-(a 6)$ be satisfied, then the solution of (1)-(3) is unique.

Proof. Let $Y_{1}$ and $Y_{2}$ be two solutions of (11), then

$$
\begin{aligned}
\left\|Y_{1}(t)-Y_{2}(t)\right\|_{2} & \leq \int_{0}^{\eta}\left\|h_{2}\left(s, Y_{2}(s)\right)-h_{2}\left(s, Y_{1}(s)\right)\right\|_{2} d s \\
& +\sqrt{\int_{0}^{t}\left\|g_{2}\left(s, I^{1-\alpha} g_{1}\left(s, Y_{1}(\phi(s))\right)\right)-g_{2}\left(s, I^{1-\alpha} g_{1}\left(s, Y_{2}(\phi(s))\right)\right)\right\|_{2}^{2} d s} \\
& \leq c T\left\|Y_{1}-Y_{2}\right\|_{C}+\frac{b^{2} T^{\frac{3}{2}-\alpha}}{\Gamma(2-\alpha)}\left\|Y_{1}-Y_{2}\right\|_{C} \\
& \leq\left(c T+b^{2} T^{2}(2-\alpha)\right)\left\|Y_{1}-Y_{2}\right\|_{C} .
\end{aligned}
$$

and

$$
\left(1-c T-b^{2} T^{2}(2-\alpha)\right)\left\|Y_{1}-Y_{2}\right\|_{C} \leq 0 .
$$

Using (a6) we can get

$$
\left\|Y_{1}-Y_{2}\right\|_{C}=0
$$

then the solution of (11) is unique. Consequently, the solution of (10) is unique.

Combining the results, then we deduce that the solution $X, Y \in C$ of the problem the problem (1)-(3) is unique.

\subsection{Continuous Dependence}

Theorem 3. The unique solution of the problem (1)-(3) depends continuously on $X_{o}, Y_{o}$.

Proof. Let $\hat{X}, \hat{Y}$ be the solution of

$$
\begin{aligned}
& \hat{X}(t)=\hat{X}_{o}-\int_{0}^{\tau} h_{1}\left(s, I^{1-\beta} g_{1}(s, \hat{Y}(\phi(s)))\right) d W(s)+\int_{0}^{t} g_{1}(s, \hat{Y}(\phi(s))) d s, \\
& \hat{Y}(t)=\hat{Y}_{o}-\int_{0}^{\eta} h_{2}(s, \hat{Y}(s)) d s+\int_{0}^{t} g_{2}\left(s, I^{1-\alpha} g_{1}(s, \hat{Y}(\phi(s)))\right) d W(s) .
\end{aligned}
$$

Let

$$
\left\|X_{o}-\hat{X}_{o}\right\|_{2} \leq \delta \text { and }\left\|Y_{o}-\hat{Y}_{o}\right\|_{2} \leq \delta
$$

then

$$
\begin{aligned}
&\|Y-\hat{Y}\|_{C}\left.\leq\left\|Y_{o}-\hat{Y}_{o}\right\|_{2}+c\|Y-\hat{Y}\|_{C} T+\frac{b^{2} T^{\frac{3}{2}-\alpha}}{\Gamma(2-\alpha)}\right)\|Y-\hat{Y}\|_{C} \\
& \leq\left\|Y_{o}-\hat{Y}_{o}\right\|_{2}+\left(c T+b^{2} T^{2}(2-\alpha)\right)\|Y-\hat{Y}\|_{C} \\
&\left(1-c T-b^{2} T^{2}(2-\alpha)\right)\|Y-\hat{Y}\|_{C} \leq\left\|Y_{0}-\hat{Y}_{0}\right\|_{2}
\end{aligned}
$$

and

$$
\|Y-\hat{Y}\|_{C} \leq \frac{\delta}{1-c T-b^{2} T^{2}(2-\alpha)}=\epsilon .
$$

In the same way, we have

$$
\begin{gathered}
\|X-\hat{X}\|_{C} \leq\left\|X_{o}-\hat{X}_{o}\right\|_{2}+\left[\frac{c T^{1-\beta} b\|Y-\hat{Y}\|_{C}}{\Gamma(2-\beta)}\right] \sqrt{T}+b T\|Y-\hat{Y}\|_{C}, \\
\|X-\hat{X}\|_{C} \leq\left\|X_{o}-\hat{X}_{o}\right\|_{2}+\left[c b T^{2}(2-\beta)+b T\right]\|Y-\hat{Y}\|_{2}
\end{gathered}
$$

and

$$
\|X-\hat{X}\|_{C} \leq \delta\left\{1+\frac{c b T^{2}(2-\beta)+b T}{1-c T-b^{2} T^{2}(2-\alpha)}\right\}=\epsilon .
$$


Theorem 4. The solution of (1)-(3) depends continuously on $h_{1}$ and $h_{2}$.

Proof. Let $\hat{X}, \hat{Y}$ be the solution of

$$
\begin{gathered}
\hat{X}(t)=X_{o}-\int_{0}^{\tau} h_{1}^{*}\left(s, I^{1-\beta} g_{1}(s, \hat{Y}(\phi(s)))\right) d W(s)+\int_{0}^{t} g_{1}(s, \hat{Y}(\phi(s))) d s, \\
\hat{Y}(t)=Y_{o}-\int_{0}^{\eta} h_{2}^{*}(s, \hat{Y}(s)) d s+\int_{0}^{t} g_{2}\left(s, I^{1-\alpha} g_{1}(s, \hat{Y}(\phi(s)))\right) d W(s), \\
\text { If }\left\|h_{j}^{*}(s, .)-h(s, .)\right\|_{2} \leq \delta_{1}, \quad j=1,2 \text { then } \\
\|Y(t)-\hat{Y}(t)\|_{2}=\| \int_{0}^{\eta}\left[h_{2}^{*}(s, \hat{Y}(s))-h_{2}(s, Y(s))\right] d s \\
+\int_{0}^{t}\left[g_{2}\left(s, I^{1-\alpha} g_{1}(s, Y(\phi(s)))\right)-g_{2}\left(s, I^{1-\alpha} g_{1}(s, \hat{Y}(\phi(s)))\right)\right] d W(s) \|_{2} \\
\leq\left(\delta_{1}+c\|Y-\hat{Y}\|_{C}\right) T+\frac{b^{2} T^{\frac{3}{2}-\alpha}}{\Gamma(2-\alpha)}\|Y-\hat{Y}\|_{C} \\
\leq \delta_{1} T+\left[c T+b^{2} T^{2}(2-\alpha)\right]\|Y-\hat{Y}\|_{C} \\
\text { and }
\end{gathered}
$$

and similarly we have

$$
\begin{aligned}
\|X(t)-\hat{X}(t)\|_{2} & \leq\left\|\int_{0}^{\eta}\left[h_{1}^{*}\left(s, I^{1-\beta} g_{1}(s, \hat{Y}(\phi(s)))\right)-h_{1}\left(s, I^{1-\beta_{g}}(s, Y(\phi(s)))\right)\right] d W(s)\right\|_{2} \\
& \left.+\| \int_{0}^{t}\left[g_{1}(s, Y(\phi(s)))\right)-g_{1}(s, \hat{Y}(\phi(s)))\right] d s \|_{2} \\
& \leq \sqrt{\int_{0}^{\tau}\left(\delta_{1}+\frac{b c T^{1-\beta}\|Y-\hat{Y}\|_{C}}{\Gamma(2-\beta)}\right)^{2} d s+b T\|Y-\hat{Y}\|_{C}} \\
& \leq\left(\delta_{1}+\frac{b c T^{1-\beta}\|Y-\hat{Y}\|_{C}}{\Gamma(2-\beta)}\right) \sqrt{T}+b T c \\
& \leq \delta_{1} \sqrt{T}+\left[b c T^{2}(2-\beta)+b T\right]\|Y-\hat{Y}\|_{C}
\end{aligned}
$$

and

$$
\|X-\hat{X}\|_{C} \leq \delta_{1} T\left[1+\frac{b c T^{2}(2-\beta)+b T}{1-c T-b^{2} T^{2}(2-\alpha)}\right]=\epsilon .
$$

From (18) and (19) the solution $X, Y$ depends continuously on $h_{1}, h_{2}$.

Theorem 5. The solution of (1)-(3) depends continuously on $D^{\alpha} X(t)=u(t)$.

Proof. Let $\hat{X}, \hat{Y}$ be the solution of

$$
\begin{aligned}
& \hat{X}(t)=X_{o}-\int_{0}^{\tau} h_{1}\left(s, I^{\alpha-\beta} \hat{u}(s)\right) d W(s)+I^{\alpha} \hat{u}(t) \\
& \left.\hat{Y}(t)=Y_{o}-\int_{0}^{\eta} h_{2}(s, \hat{Y}(s)) d s+\int_{0}^{t} g_{2}(s, \hat{u}(s))\right) d W(s)
\end{aligned}
$$

such that $\|u \hat{(t)}-u(t)\|_{2} \leq \delta_{2}$, then 


$$
\begin{aligned}
\|X(t)-\hat{X}(t)\|_{2} & \leq\left\|\int_{0}^{\eta}\left[h_{1}\left(s, I^{\alpha-\beta} \hat{u}(s)\right)-h_{1}\left(s, I^{\alpha-\beta} u(s)\right)\right] d W(s)\right\|_{2}+\left\|I^{\alpha} u(t)-I^{\alpha} \hat{u}(t)\right\|_{2} \\
& \leq \frac{c T^{\alpha-\beta} \delta_{2} \sqrt{T}}{\Gamma(\alpha-\beta+1)}+\frac{T^{\alpha} \delta_{2}}{\Gamma(\alpha+1)}
\end{aligned}
$$

and

$$
\|X-\hat{X}\|_{C} \leq T^{\alpha} \delta_{2}\left[\frac{c T^{\frac{1}{2}-\beta}}{\Gamma(\alpha-\beta+1)}+\frac{1}{\Gamma(\alpha+1)}\right]=\epsilon .
$$

By the same way

$$
\begin{aligned}
\|Y(t)-\hat{Y}(t)\|_{2} & \leq\left\|\int_{0}^{\eta}\left[h_{2}(s, \hat{Y}(s))-h_{2}(s, Y(s))\right] d s\right\|_{2}+\left\|\int_{0}^{t}\left[g_{2}(s, u(s))-g_{2}(s, \hat{u}(s))\right] d W(s)\right\|_{2} \\
& \left.\leq c T\|Y-\hat{Y}\|_{C}\right)+b \sqrt{T} \delta_{2}=\epsilon .
\end{aligned}
$$

Now

$$
\|Y-\hat{Y}\|_{C} \leq \frac{b T \delta_{2}}{(1-c T)}=\epsilon
$$

which is complete the proof.

\section{Solutions of the Problem (1)-(2) and (4)}

Let $\Lambda=C\left(I, L_{2}(\Omega)\right) \times C\left(I, L_{2}(\Omega)\right)$ be the set of ordered pairs $(X, Y), X, Y \in C$ and

$$
\|(X, Y)\|_{\Lambda}=\|X\|_{C}+\|Y\|_{C} .
$$

Define the mapping $P(X, Y)=\left(P_{1} Y, P_{2} X\right)$ where $P_{1} Y, P_{2} X$ are given by

$$
\begin{aligned}
& P_{1} Y(t)=X_{0}-\int_{0}^{\tau} h_{1}(s, Y(s)) d W(s)+\int_{0}^{t} g_{1}(s, Y(\phi(s))) d s, \\
& P_{2} X(t)=Y_{0}-\int_{0}^{\eta} h_{2}(s, X(s)) d s+\int_{0}^{t} g_{2}\left(s, I^{1-\alpha} g_{1}(s, Y(\phi(s))) d W(s) .\right.
\end{aligned}
$$

Consider the set $Q$,

$$
\left.Q_{=}\left\{(X, Y) \in L_{2}(\Omega),(X, Y) \in \Lambda:\|(X, Y)\|_{\Lambda}=\|X(t)\|_{2}+\|Y(t)\|_{2}\right\} \leq r\right\} .
$$

\subsection{Existence Theorem}

Theorem 6. Let $T \geq 1$ and (a1) - (a6) be satisfied, then (1)-(2) and (4) has a solution $(X, Y) \in \Lambda$.

Proof. Let $(X, Y) \in Q$, then we have

$$
\begin{aligned}
\left\|P_{1} Y(t)\right\|_{2} & \leq\left\|X_{0}\right\|_{2}+\left\|\int_{0}^{\eta} h_{1}(s, Y(s)) d W(s)\right\|_{2}+\left\|\int_{0}^{t} g_{1}(s, Y(\phi(s))) d s\right\|_{2} \\
& \left.\leq\left\|X_{0}\right\|_{2}+\left(K+c\|Y\|_{C}\right) \sqrt{T}+\left(M+b\|Y\|_{C}\right)\right) T \\
& \leq\left\|X_{0}\right\|_{2}+M T+K \sqrt{T}+(c \sqrt{T}+b T)\|Y\|_{C} \\
& \leq\left\|X_{0}\right\|_{2}+(M+K) T+(c+b) T\|Y\|_{C}
\end{aligned}
$$




$$
\begin{aligned}
\left\|P_{2} X(t)\right\|_{2} & \leq\left\|Y_{0}\right\|_{2}+\left\|\int_{0}^{\eta} h_{2}(s, X(s)) d s\right\|_{2}+\left\|\int_{0}^{t} g_{2}\left(s, I^{1-\alpha} g_{1}(s, Y(\phi(s)))\right) d W(s)\right\|_{2} \\
& \leq\left\|Y_{0}\right\|_{2}=\int_{0}^{\eta}\left\|h_{2}(s, X(s))\right\|_{2} d s+\sqrt{\int_{0}^{t}\left\|g_{2}\left(s, I^{1-\alpha} g_{1}(s, Y(\phi(s)))\right)\right\|_{2}^{2} d s} \\
& \leq\left\|Y_{0}\right\|_{2}+\left(K+c\|X\|_{C}\right) T+\left(M+\frac{b T^{1-\alpha}}{\Gamma(2-\alpha)}\left(M+b\|Y\|_{C}\right) \sqrt{T}\right. \\
& \leq\left\|Y_{0}\right\|_{2}+K T+M \sqrt{T}+\frac{b M T^{\frac{3}{2}-\alpha}}{\Gamma(2-\alpha)}+\frac{b^{2} T^{\frac{3}{2}-\alpha}}{\Gamma(2-\alpha)}\|Y\|_{C}+T c\|X\|_{C} \\
& \leq\left\|Y_{0}\right\|_{2}+(K+M) T+M b T^{2}(2-\alpha)+b^{2} T^{2}(2-\alpha)\|Y\|_{C}+T c\|X\|_{C} .
\end{aligned}
$$

This implies that

$$
\begin{aligned}
\|P(X, Y)\|_{X} & =\left\|\left(P_{1} Y, P_{2} X\right)\right\|_{\Lambda} \\
& =\left\|P_{1} Y\right\|_{C}+\left\|P_{2} X\right\|_{C} \\
& \leq\left\|X_{0}\right\|_{2}+\left\|Y_{0}\right\|_{2}+2(K+M) T+M b T^{2}(2-\alpha) \\
& +\left[(c+b) T+b^{2} T^{2}(2-\alpha)\right]\left\{\|X\|_{C}+\|Y\|_{C}\right\}
\end{aligned}
$$

where

$$
r=\frac{\left\|\left(X_{0}, Y_{0}\right)\right\|_{\Lambda}+2(K+M) T+M b T^{2}(2-\alpha)}{1-c T-b T-b^{2} T^{2}(2-\alpha)},
$$

then the class $\{P(X, Y)(t), \quad t=[0, T]\}$ is uniformly bounded and $P(x, y): Q \rightarrow Q$.

Let $(X, Y) \in Q, \quad t_{1}, t_{2} \in[0, T]$ such that $\left|t_{2}-t_{1}\right|<\delta$, then

$$
\begin{aligned}
\left\|P_{1} Y\left(t_{2}\right)-P_{1} Y\left(t_{1}\right)\right\|_{2} & =\left\|\int_{t_{1}}^{t_{2}} g_{1}\left(s, Y\left(\phi_{1}(s)\right)\right) d s\right\|_{2} \\
& \leq\left(M+b\|Y\|_{C}\right)\left(t_{2}-t_{1}\right), \\
\text { and } & \\
\left\|P_{2} X\left(t_{2}\right)-P_{2} X\left(t_{1}\right)\right\|_{2} & =\| \int_{t_{1}}^{t_{2}} g_{2}\left(s, I^{1-\alpha} g_{1}(s, Y(\phi(s))) d W(s) \|_{2}\right. \\
& \leq\left(M+b\left\|I^{1-\alpha} g_{1}(s, Y(\phi(s)))\right\|_{2}\right) \sqrt{\left(t_{2}-t_{1}\right)} .
\end{aligned}
$$

But

$$
\begin{aligned}
P\left(X\left(t_{2}\right), Y\left(t_{2}\right)\right)-P\left(X\left(t_{1}\right), Y\left(t_{1}\right)\right) & =\left(P_{1} Y\left(t_{2}\right), P_{2} X\left(t_{2}\right)\right)-\left(P_{1} Y\left(t_{1}\right), P_{2} Y\left(t_{1}\right)\right) \\
& =\left(\left(P_{1} Y\left(t_{2}\right)-P_{1} Y\left(t_{1}\right)\right),\left(P_{2} X\left(t_{2}\right)-P_{2} X\left(t_{1}\right)\right)\right)
\end{aligned}
$$

then from (25) and (26), $\{P(X, Y)(t)\}, t \in[0, T]$ is equicontinuous on $Q$.

Let $\left(X_{n}, Y_{n}\right) \rightarrow(X, Y) \quad$ w.p.1, $\left\{\left(X_{n}, Y_{n}\right)\right\} \in Q$ then applying stochastic Lebesgue dominated convergence Theorem [15], we can obtain 


$$
\begin{aligned}
\operatorname{L.i.m}_{n \rightarrow \infty} P\left(X_{n}, Y_{n}\right)= & \left(\text { L.i. }_{n \rightarrow \infty} P_{1} Y_{n}, \text { L.i.m } m_{n \rightarrow \infty} P_{2} X_{n}\right) \\
= & \left(\text { L.i. }_{n \rightarrow \infty}\left\{X_{0}-\int_{0}^{\tau} h_{1}\left(s, Y_{n}(s)\right) d W(s)+\int_{0}^{t} g_{1}\left(s, Y_{n}(\phi(s))\right) d s\right\},\right. \\
& \text { L.i. } \left.m_{n \rightarrow \infty}\left\{Y_{0}-\int_{0}^{\eta} h_{2}\left(s, X_{n}(s)\right) d s+\int_{0}^{t} g_{2}\left(s, I^{1-\alpha} g_{1}\left(s, Y_{n}(\phi(s))\right)\right) d W(s)\right\}\right) \\
= & \left(X_{0}-\int_{0}^{\tau} h_{1}\left(s, L . i . m_{n \rightarrow \infty} Y_{n}(s)\right) d W(s)+\int_{0}^{t} g_{1}\left(s, L . i . m_{n \rightarrow \infty} Y_{n}(\phi(s))\right)\right) d s, \\
& Y_{0}-\int_{0}^{\eta} h_{2}\left(s, L . i . m_{n \rightarrow \infty} X_{n}(s)\right) d s \\
+ & \int_{0}^{t} g_{2}\left(s, I^{1-\alpha} g_{1}\left(s, L . i . m_{n \rightarrow \infty} Y_{n}(\phi(s))\right) d W(s)\right) \\
= & \left(X_{0}-\int_{0}^{\tau} h_{1}(s, Y(s)) d W(s)+\int_{0}^{t} g_{1}(s, Y(\phi(s))) d s,\right. \\
& \left.Y_{0}-\int_{0}^{\eta} h_{2}(s, X(s)) d s+\int_{0}^{t} g_{2}\left(s, I^{1-\alpha} g_{1}(s, Y(\phi(s)))\right) d W(s),\right) \\
= & \left(P_{1} Y, P_{2} X\right)=P(x, y) .
\end{aligned}
$$

This proves that $P: Q \rightarrow Q$ is continuous.

Then the closure of $P Q$ is compact and (see [15]) the problem (1)-(2) and (4) has a solution $(X, Y) \in \Lambda, X, Y \in C\left(I, L_{2}(\Omega)\right)$.

4.2. Uniqueness of the Solution

Theorem 7. Let $\left(a^{*} 1\right)-\left(a^{*} 2\right)$ and $(a 3)-(a 6)$ are satisfied. Then the solution of $(1)-(2)$ is unique.

Proof. Let $\left(X_{1}, Y_{1}\right)$ and $\left(X_{2}, Y_{2}\right)$ be the solution of

$$
\begin{aligned}
(x(t), y(t))=\left(X_{o}\right. & -\int_{0}^{\tau} h_{1}(s, Y(s)) d W(s)+\int_{0}^{t} g_{1}(s, Y(\phi(s))) d s, \\
Y_{o} & \left.-\int_{0}^{\eta} h_{2}(s, X(s)) d s+\int_{0}^{t} g_{2}\left(s, I^{1-\alpha} g_{1}(s, Y(\phi(s)))\right) d W(s)\right),
\end{aligned}
$$

then we can get

$$
\begin{aligned}
\left\|X_{1}(t)-X_{2}(t)\right\|_{2} & \leq c \sqrt{T}\left\|Y_{1}-Y_{2}\right\|_{C}+b T\left\|Y_{1}-Y_{2}\right\|_{C} \\
& \leq(c \sqrt{T}+b T)\left\|Y_{1}-Y_{2}\right\|_{C} \\
& \leq(c+b) T\left\{\left\|X_{1}-X_{2}\right\|_{C}+\left\|Y_{1}-Y_{2}\right\|_{C}\right\} .
\end{aligned}
$$

Similarly, we can obtain

$$
\begin{aligned}
\left\|Y_{1}(t)-Y_{2}(t)\right\|_{2} & \leq c T\left\|X_{1}-X_{2}\right\|_{C}+\frac{b^{2} T^{\frac{3}{2}-\alpha}}{\Gamma(2-\alpha)}\left\|y_{1}-y_{2}\right\|_{C} \\
& \leq c T\left\|X_{1}-X_{2}\right\|_{C}+b^{2} T^{2}(2-\alpha)\left\|y_{1}-y_{2}\right\|_{C} \\
& \leq\left(c T+b^{2} T^{2}(2-\alpha)\right)\left\{\left\|X_{1}-X_{2}\right\|_{C}+\left\|Y_{1}-Y_{2}\right\|_{C}\right\} .
\end{aligned}
$$

Hence from (28) and (29)

$$
\begin{aligned}
\left\|\left(X_{1}, Y_{1}\right)-\left(X_{2}, Y_{2}\right)\right\|_{\Lambda} & =\left\|\left(X_{1}-X_{2}\right)\right\|_{C}+\left\|\left(Y_{1}-Y_{2}\right)\right\|_{C} \\
& \leq\left((b+c) T+b^{2} T^{2}(2-\alpha)\right)\left\{\left\|X_{1}-X_{2}\right\|_{C}+\left\|Y_{1}-Y_{2}\right\|_{C}\right\} \\
& \leq\left((b+c) T+b^{2} T^{2}(2-\alpha)\right)\left\|\left(X_{1}, Y_{1}\right)-\left(X_{2}, Y_{2}\right)\right\|_{\Lambda} .
\end{aligned}
$$


This implies that

$$
\left(1-(b+c) T-b^{2} T^{2}(2-\alpha)\right)\left\|\left(X_{1}, Y_{1}\right)-\left(X_{2}, Y_{2}\right)\right\|_{\Lambda} \leq 0 .
$$

then

$$
\left\|\left(X_{1}, Y_{1}\right)-\left(X_{2}, Y_{2}\right)\right\|_{\Lambda}=0
$$

and $\left(X_{1}, Y_{1}\right)=\left(X_{2}, Y_{2}\right)$.

4.3. Continuous Dependence

Theorem 8. The solution of the problem (1)-(2) and (4) is continuously dependent on $\left(X_{0}, Y_{0}\right)$.

Proof. Let $(\hat{X}, \hat{Y})$ be the solution of (1)-(2) and (4)

$$
\begin{aligned}
& \hat{X}(t)=\hat{X}_{o}-\int_{0}^{\tau} h_{1}(s, \hat{Y}(s)) d W(s)+\int_{0}^{t} g_{1}(s, \hat{Y}(\phi(s))) d s, \\
& \left.\hat{Y}(t)=\hat{Y}_{o}-\int_{0}^{\eta} h_{2}(s, \hat{X}(s)) d s+\int_{0}^{t} g_{2}\left(s, I^{1-\alpha} g_{1}(s, \hat{Y}(\phi(s)))\right) d W(s)\right)
\end{aligned}
$$

such that $\left\|\left(X_{0}, Y_{0}\right)-\left(\hat{X}_{0}, \hat{Y}_{o}\right)\right\|_{\Lambda}<\delta_{3}$. Then we have

$$
\begin{aligned}
X(t)-\hat{X}(t) & =X_{o}-\hat{X}_{o}-\int_{0}^{\tau}\left[h_{1}(s, \hat{Y}(s))-h_{1}(s, Y(s))\right] d W(s) \\
& +\int_{0}^{t}\left[g_{1}(s, Y(\phi(s)))-g_{1}(s, \hat{Y}(\phi(s)))\right] d s
\end{aligned}
$$

and

$$
\begin{aligned}
\|X(t)-\hat{X}(t)\|_{2} & \leq\left\|X_{0}-\hat{X}_{0}\right\|_{C}+c \sqrt{T}\|Y-\hat{Y}\|_{C}+b T\|Y-\hat{Y}\|_{C} \\
& \leq\left\|X_{0}-\hat{X}_{0}\right\|_{2}+(b+c) T\left\{\|X-\hat{X}\|_{C}+\|Y-\hat{Y}\|_{C}\right\} .
\end{aligned}
$$

Similarly, we deduce that

$$
\begin{aligned}
\|Y(t)-\hat{Y}(t)\|_{2} & \left.\leq\left\|Y_{0}-\hat{Y}_{0}\right\|_{2}+c T\|X-\hat{X}\|_{C}+b^{2} T^{2}(2-\alpha)\|Y-\hat{Y}\|_{C}\right\} \\
& \leq\left\|Y_{0}-\hat{Y}_{0}\right\|_{2}+\left(c T+b^{2} T^{2}(2-\alpha)\right)\left\{\|X-\hat{X}\|_{C}+\|Y-\hat{Y}\|_{C}\right\}
\end{aligned}
$$

and

$$
\begin{aligned}
\|(X, Y)-(\hat{X}, \hat{Y})\|_{\Lambda} & =\|\left(X-\hat{X}\left\|_{C}+\right\|\left(Y-\hat{Y} \|_{C}\right.\right. \\
& \leq\left\|X_{0}-\hat{X}_{0}\right\|_{2}+\left\|Y_{0}-\hat{Y}_{0}\right\|_{2} \\
& +\left((b+c) T+b^{2} T^{2}(2-\alpha)\right)\left\{\|X-\hat{X}\|_{C}+\|Y-\hat{Y}\|_{C}\right\} \\
& \leq\left\|\left(X_{0}, Y_{0}\right)-\left(\hat{X}_{0}, \hat{Y}_{0}\right)\right\|_{\Lambda} \\
& +\left((b+c) T+b^{2} T^{2}(2-\alpha)\right)\|(X, Y)-(\hat{X}, \hat{Y})\|_{\Lambda} .
\end{aligned}
$$

Hence,

$$
\|(X, Y)-(\hat{X}, \hat{Y})\|_{\Lambda} \leq \frac{\delta_{3}}{1-T(b+c)-b^{2} T^{2}(2-\alpha)}=\epsilon
$$

Theorem 9. The solution of (1)-(2) and (4) depends continuously on $h_{1}$ and $h_{2}$. 
Proof. Let $(\hat{X}, \hat{Y})$ be the solution of (1)-(2) and (4) such that

$$
\begin{aligned}
& \hat{X}(t)=X_{0}-\int_{0}^{\tau} h_{1}^{*}(s, \hat{Y}(s)) d W(s)+\int_{0}^{t} g_{1}(s, \hat{Y}(\phi(s))) d s, \\
& \left.\hat{Y}(t)=Y_{0}-\int_{0}^{\eta} h_{2}^{*}(s, \hat{X}(s)) d s+\int_{0}^{t} g_{2}\left(s, I^{1-\alpha} g_{1}(s, \hat{Y}(\phi(s)))\right) d W(s)\right) . \\
& \|X(t)-\hat{X}(t)\|_{2}=\left\|\int_{0}^{\tau}\left[h_{1}^{*}(s, \hat{Y}(s))-h_{1}(s, Y(s))\right] d W(s)+\int_{0}^{t}\left[g_{1}(s, Y(\phi(s)))-g_{1}(s, \hat{Y}(\phi(s)))\right] d s\right\|_{2} \\
& \leq \sqrt{\int_{0}^{\tau}\left\|h_{1}^{*}(s, \hat{Y}(s))-h_{1}(s, Y(s))\right\|_{2}^{2} d s}+\int_{0}^{t}\left\|g_{1}(s, Y(\phi(s)))-g_{1}(s, \hat{Y}(\phi(s)))\right\|_{2} d s \\
& \leq \sqrt{\int_{0}^{\tau}\left[\left\|h_{1}^{*}(s, \hat{Y}(s))-h_{1}^{*}(s, Y(s))\right\|_{2}+\left\|h_{1}^{*}(s, Y(s))-h_{1}(s, Y(s))\right\|_{2}\right]^{2} d s} \\
& +\int_{0}^{t}\left\|g_{1}(s, Y(\phi(s)))-g_{1}(s, \hat{Y}(\phi(s)))\right\|_{2} d s \\
& \leq \sqrt{\int_{0}^{\tau}\left(c\|Y(s)-\hat{Y}(s)\|_{2}+\delta_{4}\right)^{2} d s}+\int_{0}^{t} b\|Y(s)-\hat{Y}(s)\|_{2} d s \\
& \leq(c+b) T\|Y-\hat{Y}\|_{C}+\delta_{4} T \text {. }
\end{aligned}
$$

Similarly we can get

$$
\begin{aligned}
\|Y-\hat{Y}\|_{C} & \leq c T\|X-\hat{X}\|_{C}+\frac{b^{2} T^{\frac{3}{2}-\alpha}}{\Gamma(2-\alpha)}\|Y-\hat{Y}\|_{C}+\delta_{4} T \\
& \leq c T\|X-\hat{X}\|_{C}+b^{2} T^{2}(2-\alpha)\|Y-\hat{Y}\|_{C}+\delta_{4} T
\end{aligned}
$$

and

$$
\begin{aligned}
\|(X, Y)-(\hat{X}, \hat{Y})\|_{\Lambda} & =\|\left(X-\hat{X}\left\|_{C}+\right\|\left(Y-\hat{Y} \|_{C}\right.\right. \\
& \leq 2 \delta_{4} T+c T\|X-\hat{X}\|_{C}+\left(c T+b T+b^{2} T^{2}(2-\alpha)\|Y-\hat{Y}\|_{C}\right. \\
& \leq 2 \delta_{4} T+\left[(b+c) T+b^{2} T^{2}(2-\alpha)\right]\left\{\|Y-\hat{Y}\|_{C}+\|X-\hat{X}\|_{C}\right\} \\
& \leq 2 \delta_{4} T+\left[(b+c) T+b^{2} T^{2}(2-\alpha)\right]\|(X, Y)-(\hat{X}, \hat{Y})\|_{\Lambda} .
\end{aligned}
$$

This implies that

$$
\|(X, Y)-(\hat{X}, \hat{Y})\|_{\Lambda} \leq \frac{2 \delta_{4} T}{1-(b+c) T-b^{2} T^{2}(2-\alpha)}=\epsilon
$$

which completes the proof.

Theorem 10. The solution $(X, Y)$ of (1)-(2) and (4) depends continuously on $u(t)=D^{\alpha} X(t)$, $\alpha \in(0,1]$.

Proof. Let $(\hat{X}, \hat{Y})$ be the solution of

$$
\begin{aligned}
& \hat{X}(t)=X_{o}-\int_{0}^{\tau} h_{1}(s, \hat{Y}(s)) d W(s)+I^{\alpha} \hat{u}(t), \\
& \left.\hat{Y}(t)=Y_{o}-\int_{0}^{\eta} h_{2}(s, \hat{X}(s)) d s+\int_{0}^{t} g_{2}(s, \hat{u}(s))\right) d W(s)
\end{aligned}
$$

such that $\| u \hat{u} t)-u(t) \|_{2} \leq \delta_{5}$, then 


$$
\begin{aligned}
\|(X, Y)-(\hat{X}, \hat{Y})\|_{\Lambda} & =\|\left(X-\hat{X}\left\|_{C}+\right\|\left(Y-\hat{Y} \|_{C}\right.\right. \\
& \leq\left\|\int_{0}^{\eta}\left[h_{1}(s, \hat{Y}(s))-h_{1}(s, Y(s))\right] d W(s)\right\|_{2}+\left\|I^{\alpha} u(t)-I^{\alpha} \hat{u}(t)\right\|_{2} \\
& +\left\|\int_{0}^{\eta}\left[h_{2}(s, \hat{X}(s))-h_{2}(s, X(s))\right] d s\right\|_{2}+\left\|\int_{0}^{t}\left[g_{2}(s, u(s))-g_{2}(s, \hat{u}(s))\right] d W(s)\right\|_{2} \\
& \leq c \sqrt{T}\|Y-\hat{Y}\|_{C}+\frac{T^{\alpha} \delta_{5}}{\Gamma(\alpha+1)}+c T\|X-\hat{X}\|_{C}+b \sqrt{T} \delta_{5} \\
& \leq\left(\frac{T^{\alpha}}{\Gamma(\alpha+1)}+b T\right) \delta_{5}+c T\|(X, Y)-(\hat{X}, \hat{Y})\|_{\Lambda} .
\end{aligned}
$$

Now

$$
\|(X, Y)-(\hat{X}, \hat{Y})\|_{\Lambda} \leq \frac{\delta_{5}}{1-c T}\left(\frac{T^{\alpha}}{\Gamma(\alpha+1)}+b T\right)
$$

which completes the proof.

\section{Example}

Consider the coupled system

$$
\begin{aligned}
& \frac{d X}{d t}(t)=\frac{1-t^{2}+Y(t)}{30\left(1+\|Y(t)\|_{2}\right)}, \quad t \in(0,1] \\
& d Y(t)=\frac{e^{-t}+D^{\frac{1}{2}} X(t)}{120\left(1+\|X(t)\|_{2}\right)} d W(t), \quad t \in(0,1]
\end{aligned}
$$

subject to

$$
X_{0}=\int_{0}^{\tau} \frac{s X(s)}{80+s^{2}} d W(s), \quad Y_{0}=\int_{0}^{\eta} \frac{Y(s)}{\sqrt{s+36}} d s
$$

where

$$
\left\|g_{1}(t, Y(t))\right\|_{2} \leq \frac{1}{30}\|Y(t)\|_{2}, \quad\left\|g_{2}(t, X(t))\right\|_{2} \leq \frac{1}{120}\left[1+\|X(t)\|_{2}\right]
$$

and

$$
\| h_{1}\left(t, X(t)\left\|_{2} \leq \frac{\|X(t)\|_{2}}{80}, \quad\right\| h_{2}\left(t, Y(t) \|_{2} \leq \frac{\|Y(t)\|_{2}}{6} .\right.\right.
$$

Easily, the coupled system (31) with nonlocal integral conditions (32) satisfies all the assumptions (a1)-(a6) of Theorem 1. with $b=\frac{1}{30}, c=\frac{1}{6}$.

\section{Conclusions}

Here, we have combined between the three senses of derivatives, the stochastic Itodifferential and the fractional and integer orders derivatives for the second order stochastic process in two non-local problems of the coupled system of the two random and stochastic differential Equations (1) and (2) with the stochastic and random integral conditions (3) and the coupled system of the two stochastic and random nonlocal integral conditions (4) where $X_{0}$ and $Y_{0}$ are two second order random variables.

The existence of solutions $X, Y \in C\left(I, L_{2}(\Omega)\right)$ of the problems (1)-(3) and (1)-(2) and (4) are proved. The unique solution and the sufficient conditions are discussed. The continuous dependence of the solution $X, Y \in C\left(I, L_{2}(\Omega)\right)$ on the two random variables $X_{0}$ and $Y_{0}$ and on the two random functions $h_{1}$ and $h_{2}$ and the continuous dependence of the solution $Y \in C\left(I, L_{2}(\Omega)\right)$ on the fractional order derivative $D^{\alpha} X(t)$ are be studied. An example is given. 
Author Contributions: Conceptualization, A.M.A.E.-S. and H.A.F. All authors have read and agreed to the published version of the manuscript.

Funding: This research received no external funding.

Institutional Review Board Statement: Not applicable.

Informed Consent Statement: Not applicable.

Data Availability Statement: Not applicable.

Acknowledgments: Authors are grateful to referees for their useful comments and remarks that helped to improve this work.

Conflicts of Interest: The authors declare no conflict of interest.

\section{References}

1. Soong, T.T. Random Differential Equations in Science and Engineering; Academic Press: New York, NY, USA, 1973.

2. Tsokos, C.P.; Padgett, W.J. Stochastic integral equations in life science and engineering. Int. Stat. Rev. 1973, 412, 15-38. [CrossRef]

3. Krylov, N.V. On Itô's stochastic integral equations. Theory Probabil. Its Appl. 1967, 14, 330-336. [CrossRef]

4. Adomian, G. Coupled nonlinear stochastic differential equations. J. Math. Anal. Appl. 1983, 92, 427-434. [CrossRef]

5. Bougoffa, L. A class of second order differential equations with nonlocal boundary conditions. Appl. Math. Sci. 2007, 1, 1035-1039.

6. El-Sayed, A.M.A.; Hashem, H.H.G. A coupled System of integral equations in reflexive Banach spaces. Acta Math. Sci. 2012, 32B, 2021-2028. [CrossRef]

7. El-Sayed, A.M.A.; Gaafar, F.; El-Gendy, M. Continuous dependence of the solution of Ito stochastic differential equation with nonlocal conditions. Appl. Math. Sci. 2016, 10, 1971-1982. [CrossRef]

8. El-Sayed, A.M.A.; Gaafar, F.; El-Gendy, M. Continuous dependence of the solution of random fractional-order differential equation with nonlocal condition. Fract. Diff. Calcul. 2017, 7, 135-149. [CrossRef]

9. Bashir, A.; Sotiris, K.N.; Ahmed, A. On a coupled system of fractional differential equations with coupled nonlocal and integral boundary conditions. Chaos Solitons Fractals 2016, 83, 224-234.

10. Elborai, M.M.; Abdou, M.A.; Youssef, M.I. On some nonlocal perturbed random fractional integro-differential equations. Life Sci. J. 2013, 10, 1601-1609.

11. Balasubramaniam, P.; Tamilalagan, P. The solvability and optimal controls for impulsive fractional stochastic integro-differential equations via resolvent Operators. J. Optim. Theory Appl. 2017, 174, 139-155. [CrossRef]

12. Mohamed, I.Y. On the solvability of a general class of a coupled system of stochastic functional integral equations. Arab J. Basic Appl. Sci. 2020, 27, 142-148. [CrossRef]

13. Watanabe, S.; Yamada, T. On uniqueness of solutions of stochastic differential equations. J. Math. Kyoto Univ. 1971, 11, 553-563. [CrossRef]

14. Hafez, F.M. The Fractional calculus for some stochastic processes. Stochastic Anal. Appl. 2004, 22, 507-523. [CrossRef]

15. Curtain, R.F.; Pritchard, A.J. Functional Analysis in Modern Applied Mathematics; Cambride Press: Cambride, MA, USA, 1977.

16. El-Sayed, A.M.A. On the stochastic fractional calculus operators. J. Fract. Calcul. Appl. 2015, 6, 101-109.

17. El-Sayed, A.M.A.; Hoda, A.F. On a coupled system of random and stochastic nonlinear differential equations with coupled nonlocal random and stochastic nonlinear integral conditions. Mathematics 2021, 9, 2111. [CrossRef]

18. Wong, E. Introduction to Random Processes; Springer: Berlin, Germany, 1980. 